\title{
Does sequential balloon expulsion test improve the screening of defecation disorders?
}

\author{
A. C. Caetano $0^{1,2,3^{*}}$ (B) D. Costa ${ }^{1,2,3}$, R. Gonçalves ${ }^{1}$, J. Correia-Pinto ${ }^{2,3}$ and C. Rolanda ${ }^{1,2,3}$
}

\begin{abstract}
Background: A defecation disorder (DD) is a difficulty in evacuation documented by physiological exams. However, this physiological evaluation can be cumbersome, inaccessible and costly. Three "low-cost" tools to evaluate DD_a clinical DD score, the balloon expulsion test (BET) and a digital rectal examination (DRE) score were evaluated as separate or combined tests for DD screening.

Methods: This prospective study occurred between January 2015 and March 2019 in the Gastroenterology Department of a tertiary hospital. Besides the gold standard physiological tests, constipated patients answered the clinical DD score and were evaluated by DRE and BET [standard and variable volume (VV)].

Results: From 98 constipated patients, 35 (38.9\%) were diagnosed with DD according to Rome IV criteria, mainly female $(n=30,86 \%)$ with a median age of 60 years old. The clinical DD score revealed an AUC of 0.417 (SE $=0.07$, $p=0.191)$. The DRE score displayed an AUC of $0.56(\mathrm{SE}=0.063, p=0.301)$. The standard BET displayed a sensitivity of $86 \%$, specificity of $58 \%$, positive predictive value (PPV) of $57 \%$ and negative predictive value (NPV) of $86 \%$. The sequential VBET followed by standard BET improved the BET performance regarding the evaluation of DD, with a sensitivity of $86 \%$, specificity of $67 \%$, PPV of $63 \%$ and NPV of $87 \%$. The sequential BET had an OR $8.942, p>0.001, \mathrm{Cl}$ 3.18-25.14, revealing to be the most significant predictor for DD screening.
\end{abstract}

Conclusion: The sequential BET is a low cost, well-performing DD screening tool, appropriate to the Primary Care Setting.

Keywords: Constipation, Defecation disorders, Low-cost tools

\section{Background}

A defecation disorder (DD) is defined as a difficulty in evacuation or emptying the rectum. DD may result from impaired anorectal function or rectal structural disturbances in patients with complaints of Chronic Constipation $(\mathrm{CC})[1-3]$.

DD was recently defined by the Rome IV criteria, based on symptoms and objective physiological criteria-Table $1[4,5]$. Therefore, the diagnosis of $\mathrm{DD}$ is established when a patient with functional chronic

\footnotetext{
*Correspondence: anaceliacaetanocs@gmail.com

1 Department of Gastroenterology, Hospital of Braga, Sete Fontes - São Victor, 4710-243 Braga, Portugal

Full list of author information is available at the end of the article
}

constipation (CC) or irritable bowel syndrome with constipation (IBS-C) has impaired evacuation as demonstrated by 2 of 3 types of tests-balloon expulsion test (BET); imaging (conventional defecography, dynamic ultrasound or dynamic magnetic resonance); anorectal manometry (ARM) or electromiography (EMG). This physiological evaluation is not always easily accessible, moreover it can be long and costly $[3,6,7]$. American studies report costs of healthcare utilization for CC as high as 500 dollars-patient-year while the exact impact of CC diagnostic assessment and treatment in Western Europe healthcare systems is unknown [8].

Thus, there is a subgroup of constipated patients-with DD-that can benefit from specific treatment behind laxatives, and we do not want them to miss proper treatment 
Table 1 Rome IV diagnostic criteria for functional defecation disorders

Rome IV Diagnostica criteria (a) for functional defecation disorders

1. The patient must satisfy diagnostic criteria for functional constipation and /or Irritable Bowel Syndrome with constipation

2. During repeated attempts to defecate, there must be features of impaired evacuation, as demonstrated by 2 of the 3 following 3 tests: a. Abnormal balloon expulsion test

b. Abnormal anorectal evacuation pattern with manometry or anal surface electromyography

c. Impaired rectal evacuation by imaging

(a) Criteria fulfilled for the last 3 months with symptoms onset at least 6 months before diagnosis

Table 2 Renzi clinical DEFECATION DISORDERS Score

\begin{tabular}{llllll}
\hline Symptoms/score & $\mathbf{0}$ & $\mathbf{1}$ & $\mathbf{2}$ & $\mathbf{3}$ & $\mathbf{4}$ \\
\hline $\begin{array}{l}\text { Excessive straining } \\
\begin{array}{l}\text { Incomplete rectal evacu- } \\
\text { ation }\end{array}\end{array}$ & Never & Rarely & Sometimes & Usually & Always \\
$\begin{array}{l}\text { Use of enemas/laxatives } \\
\begin{array}{l}\text { Vaginal/Perineal digital } \\
\text { pressure }\end{array}\end{array}$ & Never & Rarely & Sometimes & Usually & Always \\
$\begin{array}{c}\text { Abdominal discomfort/ } \\
\text { pain }\end{array}$ & Never & Rarely & Sometimes & Usually & Always \\
\hline
\end{tabular}

Never, never; rarely, $<1 /$ month; sometimes, $<1 /$ week, $\geq 1$ /month; usually, $<1 /$ day; $\geq 1$ week; always, $\geq 1$ /day

in consequence of the DD underdiagnosis. However, with the nowadays cost-effectiveness constraints, it may be impossible to perform the recommended physiological evaluation to all patients seeking a medical consultation for CC or IBS-C. A cheaper but satisfactory screening approach of DD that promotes an adequate selection of complementary tests and an earlier and adequate treatment seems ideal. Applying the creative concept of "lowcost" tools, we run an extensive review of the subject [9]. We found 3 potential "low-cost" tools-a DD clinical score, the BET and the digital rectal examination (DRE). Based on current evidence, it is not possible to know whether these "low-cost" strategies are useful in this setting [9].

Going into detail, no clinical score has emerged as a relevant diagnostic method in the diagram of DD and none was evaluated as a screening tool. Two specific DD scores (Altomare score and Renzi score) were validated to grade severity and value of treatment's efficacy [10, 11]. Two important limitations prevented us from using Altomare score-it includes "time spent at the toilet" and "stool consistency", items with a strong cultural influence and diet effect. The Renzi score is a 5-items score (Table 2) that assesses various complains of an abnormal evacuation and shows less cultural impact. The BET is a convenient procedure but described with inconsistent methodology especially regarding the volume used to inflate the balloon $[9,12-14]$. Considering the physiological aspects of defecation, there remains doubts if a fixed low intrarectal volume is enough to trigger the desire to defecate or if higher variable volumes associated with the constant desire to evacuate can compensate for rectal hiposensitivity $[2,12]$. The DRE is another lowcost tool that may sometimes be under or inappropriately used [15-17] and its value in DD was assessed in very few studies [9]. Only Tantiphlachiva proposed a DRE score to diagnose DD [18]. Therefore, there is a need for prospective studies with descriptive and consistent methodology to evaluate BET and DRE utility. In addition, it seems crucial an external validation of the few studies published so far $[11,12,18]$. Furthermore, no study in the literature evaluated a clinical DD score (or combination of its individual items) and these "low-cost" physiological tests-BET and DRE score. This effort seems relevant in the screening of DD in primary health care but also with potential utility in the diagnosis and assessment of treatment's efficacy. This strategy may generate clear clinical and financial advantages $[1,2]$. The aim of this study was to evaluate the performance of the "low-cost" tests in the screening of DD as separate or combined tools.

\section{Methods}

Study design and subjects

This was a cross-sectional study. Consecutive patients with CC or IBS-C (Rome IV criteria), followed in the Department of Gastroenterology of Braga Hospital between January 2015 and March 2019 were prospectively proposed to the study protocol. The exclusion criteria were previous colonic and anorectal surgery, inflammatory bowel disease, colorectal cancer, anal cancer, other secondary causes of constipation, anorectal abnormalities that would influence symptoms of defecation detected by proctologic examination as anal fissure and haemorrhoids, incapacity to understand the study protocol.

During the clinical interview, the Renzi DD score was applied, followed by the DRE and the BET protocol, always by the same operator, as described in the next sections. The gold standard physiological tests (in our department anorectal manometry and defecation 
imaging) were subsequently scheduled and performed by another operator blinded to the study. A positive diagnosis of DD was considered according to 2 criteria of Rome IV, excluding information from the BET. Our constipated patients with no DD according with the current standards of diagnosis served as control group. Thus, patients with a positive BET plus a positive ARM or defecography were not included in DD group and were excluded from the control group (NoDD).

The Ethics Committee for Health of the Hospital of Braga approved the research protocol. Written informed consent was obtained from all participants. All data were collected anonymously and Portuguese regulations applicable to the management of personal data were followed at all times.

\section{Renzi DD score}

A clinical DD score developed and validated by Renzi, as described in introduction and shown in Table 2, is divided in 5 items-excessive straining, incomplete rectal evacuation, use of enemas/laxative, vaginal/perineal digital pressure, abdominal discomfort/pain-that are scored from 0 to 4 points according to its frequency in everyday patients' life. A final score $\geq 9$ points was assumed as abnormal [11]. We validated the Portuguese version of the score [19] according to the Consensus based Standards for the selection of the Health Measurement Instruments (COSMIN) checklist [20] and following guidelines for cross-cultural validity [21]. The score was applied in a face to face interview. In this study, the score was also deconstructed and evaluated in diverse combinations of items.

\section{DRE technique}

The general DRE was performed according to the technique described by Talley [15]. It was followed by the specific tests for pelvic floor dysfunction: the patient was requested to strain and try to push out the finger (to assess paradoxical external anal sphincter and puborectalis contraction), then the patients was asked regarding pain when pressing the posterior rectal wall (to assess puborectalis muscle tenderness) and then a hand was placed on the anterior abdominal wall of the patient while asking him/her to strain again (to assess if the patient is excessively contracting the abdominal wall). The DRE was performed always by the same examiner (the main investigator) who knew the patients previously from the outpatient consultation. Next, we applied the Tanthiplachiva score defining a positive diagnosis of DD when two of the following criteria were present: (1) paradoxical anal contraction or impaired anal relaxation, (2) impaired push effort, (3) absence of perineal descent [18].

\section{BET technique}

With the patient lying in the left lateral position, an empty $4 \mathrm{~cm}$ long balloon covered with lubricating jelly and tied to a flexible catheter (external diameter, $6 \mathrm{~mm}$ ) was placed in the rectum. The balloon was then filled with $50 \mathrm{ml}$ of air through the catheter. The patient was asked to expel the balloon. A stop-watch was started and stopped when the patient expelled the device. The time taken for expelling the balloon was recorded. An abnormal BET (standard BET) was defined as inability to expel the balloon in less than $1 \mathrm{~min}$. A second BET was performed following the same steps but with a variable volume of air-the volume of air associated with a constant desire to evacuate (vvBET).

\section{Statistical evaluation}

Continuous data is presented as median and interquartile range. Normal distribution was checked using skewness and kurtosis. Comparisons among groups were carried out using the Chi-square test or Mann-Whitney test. The ROC curves were used to evaluate the performance of the continuous variables (clinical DD score and DRE score). Sensitivity and specificity were calculated for the abnormal clinical score, altered DRE, abnormal standard BET, abnormal vvBET and abnormal sequential BET. Candidate variables for inclusion in a prediction model were any significant (or borderline significant) variables at univariate analysis or variables whose inclusion was supported by the existing literature. Potential predictors were identified using backward stepwise selection. $p$ values $<0.05$ was defined for rejection of the null hypothesis. All the statistical analyses were conducted using the software SPSS 22.0 (IBM, USA).

\section{Results}

From 98 patients with the clinical criteria of CC, 8 were excluded because there was not enough data to admit or exclude the DD or NoDD diagnosis. Thirty-five patients (38.9\%) were diagnosed with DD, mainly female $(n=30$, $86 \%)$ with a median age of 60 years old. The 55 constipated patients without criteria for DD (NoDD) were also mainly female $(\mathrm{n}=51,93 \%)$ but with a median age of 51 years old $(p=0.009)$. Table 3 describes demographic and clinical characteristics of the patients.

\section{Renzi DD score}

Regarding clinical complaints, excessive straining, was reported as usually or always by $64.7 \%$ of the DD patients and by $63.6 \%$ of the NoDD patients. Only 3 DD patients refer never perform excessive straining in evacuation and no patient of the NoDD group refer never perform excessive straining. Regarding 
Table 3 Patients demographic and clinical characteristics

\begin{tabular}{llll}
\hline Characteristics & DD group & NoDD group & $\boldsymbol{p}$ \\
\hline Age (years) & 60 & 51 & 0.009 \\
Gender (fem/male) & $30 / 5$ & $51 / 4$ & 0.28 \\
Total Renzi score & 11 & 11 & 0.452 \\
ARM rest pressure $(\mathrm{mmHg})$ & 62 & 63 & 0.792 \\
ARM sustained pressure $(\mathrm{mmHg})$ & 101 & 105 & 0.805 \\
ARM minimal sensitivity $(\mathrm{ml})$ & 97 & 96 & 0.991 \\
ARM sustained sensitivity $(\mathrm{ml})$ & 203 & 192 & 0.668 \\
ARM maximal sensitivity $(\mathrm{ml})$ & 242 & 221 & 0.302 \\
Presence of anal defects & 3 & 2 & \\
Presence of structural abnormalities & 18 & 12 & \\
\hline
\end{tabular}

ARM anorectal manometry

incomplete rectal evacuation, $61.8 \%$ of the DD patients and $69 \%$ of the NoDD patients complain to feel it usually or always. Again only 2 patients in the DD group and 1 patient in the NoDD group describe never feel incomplete rectal evacuation. Regarding the use of enemas or laxatives, $48.5 \%$ of the DD patients refer to use it usually or always as well as $56.3 \%$ of the NoDD patients. Only $14.5 \%$ of the NoDD patients and $27.3 \%$ of the DD patients refer never use it. The vaginal/perineal digital pressure was usually or always used by $29.4 \%$ of the DD patients and by $16.3 \%$ of the NoDD patients; $52.9 \%$ of the DD patients and $43.9 \%$ of the NoDD patients refer never to require it. Abdominal discomfort or pain was felt usually or always by $34 \%$ of the DD patients and by $40 \%$ of the NoDD patients. Four patients in both groups never felt abdominal pain.

The clinical DD score was abnormal in $89 \%$ of the NoDD patients and in $64.7 \%$ of patients with DD $(p=0.04)$. Regarding each individualized item of the clinical DD score there were no significant differences between the groups $(p>0.05)$. The abnormal clinical DD score (score $\geq 9$ points) displayed a sensitivity of $65 \%$, specificity of $10 \%$, positive predictive value (PPV) of $31 \%$ and negative predictive value (NPV) of $33 \%$. It revealed an AUC of $0.417(\mathrm{SE}=0.07, p=0.191)$.

\section{DRE technique}

Regarding the DRE, paradoxical anal contraction or impaired anal relaxation was identified in $31.4 \%$ of the DD patients and in $16.3 \%$ of the NoDD patients $(p=0.094)$. Impaired push effort was recognized in $34.3 \%$ of the DD patients and in $23.6 \%$ of the NoDD patients $(p=0.272)$. The absence of perineal descent was documented in $28.6 \%$ of the DD patients and in $27.3 \%$ of the NoDD patients $(p=0.893)$.

The DRE was abnormal in $18.2 \%$ of the NoDD patients and in $28.6 \%$ of DD patients $(p=0.248)$. The abnormal clinical DD score displayed a sensitivity of $29 \%$, specificity of $82 \%$, PPV of $50 \%$ and NPV of $64 \%$. The DRE score displayed an AUC of $0.56(\mathrm{SE}=0.063, p=0.301)$.

\section{BET technique}

The standard BET was abnormal in $41.8 \%$ of the NoDD patients and in $85.7 \%$ of DD patients $(p<0.001)$. Evaluating the vvBET, the median volume of the BET associated with a constant desire to evacuate was $133.2 \pm 60.9 \mathrm{ml}$. The vvBET was abnormal in $32.7 \%$ of the NoDD patients and in $82.9 \%$ of DD patients $(p<0.001)$. The performance of the BET using fixed volume and variable volume was different in 6 patients -5 patients were capable of expelling the variable-volume balloon (normal vvBET) but not the fixed-volume balloon (abnormal standard BET) and one patient had the inverse performance.

The standard BET displayed a sensitivity of $86 \%$, specificity of $58 \%$, PPV of $57 \%$ and NPV of $86 \%$. The vvBET alone showed a sensitivity of $83 \%$, specificity of $67 \%$, PPV of $62 \%$ and NPV of $86 \%$. The sequential BET (vvBET followed by standard BET) improves the BET performance regarding evaluation of $\mathrm{DD}$, with a sensitivity of $86 \%$, specificity of $67 \%$, PPV of $63 \%$ and NPV of $88 \%$.

\section{Tool to screen DD}

Table 4 displays the performing characteristics of the "low-cost" tools under evaluation.

At univariate analysis, only age $(p=0.022)$, standard BET $(p<0.001)$, vvBET $(p<0.001)$ and sequential BET $(p<0.001)$ were significant predictors of DD. Logistic regression demonstrated that sequential BET had an

Table 4 Performance of the low-cost tools

\begin{tabular}{|c|c|c|c|c|c|}
\hline Tool /performance measurement & Sensitivity & Specificity & PPV & NPV & Accuracy \\
\hline Abnormal (>9 points) clinical DD score & 65 & 10 & 31 & 33 & 31 \\
\hline Abnormal ( $\geq 2$ points) Digital rectal examination & 29 & 82 & 50 & 64 & 61 \\
\hline FV BET (standard) & 86 & 58 & 57 & 86 & 69 \\
\hline W BET & 83 & 67 & 62 & 86 & 73 \\
\hline Sequential BET ( $\mathrm{W} » F V)$ & 86 & 67 & 63 & 88 & 75 \\
\hline
\end{tabular}

$D D$ defecation disorder, $B E T$ balloon expulsion test, $V V$ variable volume, $F V$ fixed volume, $P P V$ positive predictive value, $N P V$ negative predictive value 
OR 8.942, $p>0.001$, CI 3.18-25.14 and that the sequential BET standed out as the most significant predictor for screening DD.

\section{Discussion}

Chronic constipation is one of the five most common gastrointestinal disorders. It consumes substantial health care resources due to the high prevalence and specificity of the diagnostic tests and treatments involved $[3,7]$. In the current times, with financial cutbacks in healthcare, the judicious use of technology seems to be a relevant issue $[7,22]$. Taking all these aspects in consideration, we selected 3 "low-cost" tools in order to understand their role in the screening of DD as potential tools to be used in a first approach, namely in Primary Care Setting.

As shown in Table 3, demographic and clinical characteristics of both DD patients and NoDD patients are identical except for age as DD patients are significantly older (60 vs 51 years old), that is also reported in other series and can be explained by the cumulative structural and physiological alterations in the pelvic floor of older women. $\left({ }^{1-3,6,23}\right)$.

The Renzi clinical DD score, in the original study, showed good discriminatory power to distinguish between patients and controls (sensitivity $92 \%$ and specificity $96 \%$ ) and also variations in patients over time [11]. However, in our study population, the clinical score did not perform well as a screening tool (sensitivity $65 \%$ and specificity 10\%). In fact, Renzi et al. validated their score specifically to grade defecation disorders and not as a diagnostic tool among constipated patients. Besides their patients' sample was selected according to Rome III criteria and specific exclusion criteria-no irritable bowel syndrome and no slow transit constipation. That way it is difficult to reproduce their good results using their score as a screening tool. This goes in line with recent reviews that consider clinical scores useless for screening or diagnostic purposes in DD [24, 25]. One possible explanation is that patients, when asked about their symptoms, tend to exaggerate their complains when evoking them retrospectively. No item of the clinical DD score had a distinctive individualized performance, not even the most controversial item of the DD clinical score-"abdominal discomfort/pain"-pointing to the continuous spectrum of pain in the DD subgroup of these pathologies (IBS with constipation and CC).

The DRE score, similarly, did not perform well as a screening tool. Compared with the results presented by Tantiphlachiva in their sample of 209 patients (sensitivity of $75 \%$ and specificity of $87 \%$ ), the DRE score had a poorer performance in our study population (sensitivity $29 \%$ and specificity $82 \%$ ) [18]. The DRE is an operatordependent technique. Although we tried to decrease this bias with the execution of the DRE always by the same operator, we still have to take into account the years of professional experience of the main investigator (5 years) compared with the Tantiphlachiva team. It would be interesting to evaluate the learning curve of the DRE technique. Another possible bias is the cultural barrierit is a dynamic evaluation, and different populations may not consistently perform the same oral instructions.

The standard BET performance (evaluation of the ability to evacuate solid stool) is in accordance with the majority of the data presented in the literature [9]. Trying to discriminate the best performance of the BET, besides the standard BET, we evaluated the vvBET (evaluation of rectal sensory function, which can also disturb evacuation ability). The sequential BET, where vvBET is followed by standard BET, improved the BET performance regarding evaluation of $\mathrm{DD}$. These results points to the importance of rectal filling and its conscious awareness for a correct BET, improving the BET capability as a screening tool. Our results validate Minguez et al. results [12]. We also shared their enthusiasm that simple tools can be easily performed in any examination room and can be incorporated in the preliminary evaluation of patients with CC. The sequential BET increases specificity, PPV and NPV to this tool. Increasing age can also add specificity to the BET sequence. So, the sequential BET proposed could become an interesting tool for screening constipated patients in the Primary Care setting.

This study has some limitations already pointed outcultural barrier regarding the patients, years of experience regarding the investigators. The left lateral position to perform the BET can also be seen as a limitation as the sitting position is more physiological [26]. The sample size can also be seen as a limitation. In our defense, while both the clinical score and the DRE score did not reveal discriminative power to screen constipated patients, the standard BET had a similar performance to that reported by other series [9]. Besides, as we know, an algorithm or score always performs better in the validation population (reported in the original papers) and the consistency of the results when performing the external validation is often not achieved. Pursuing the refinement of the lowcost tools, the sequential BET seems the most suitable to potential use in the Primary Care Setting.

\section{Conclusion}

The clinical DD score and the DRE did not reveal discriminative power to evaluate patients with DD. The BET stands as a good, reproducible and low-cost tool, that performs better when sequentially used with variable volume and fixed volume. Age can improve the BET specificity to exclude DD. 


\section{Abbreviations}

AUC: Area under the curve; ARM: Anorectal manometry; BET: Balloon expulsion test; CC: Chronic constipation; DRE: Digital rectal examination; DD: Defecation disorder; EMG: Electromiography; IBS-C: Irritable bowel syndromesubtype constipation; ROC: Receiver Operating Characteristic; W: Variable volume; PPV: Positive predictive value; NPV: Negative predictive value.

\section{Acknowledgements}

Not applicable.

\section{Authors' contributions}

ACC and CR were involved in the study concept and design; ACC and DC were involved in the acquisition of data and completed the analysis and interpretation of data; DC and RG reviewed the statistical analysis; ACC, DC and CR contributed to the drafting of the manuscript; JCP and CR made a critical revision and English editing of the manuscript. At the end of the process, all authors have read and approved the manuscript.

\section{Funding}

No funding sources were used.

\section{Availability of data and materials}

The data is available from the corresponding author in digital format that can be sent to the journal.

\section{Ethics approval and consent to participate}

The Ethics Committee for Health of the Hospital of Braga approved the research protocol. Written informed consent was obtained from all participants. All data were collected anonymously and Portuguese regulations applicable to the management of personal data were followed at all times.

\section{Consent to publish}

The authors give their consent to publish.

\section{Competing interests}

We declare there is no conflict of interest or disclosure to formulate.

\section{Author details}

1 Department of Gastroenterology, Hospital of Braga, Sete Fontes - São Victor, 4710-243 Braga, Portugal. ${ }^{2}$ Life and Health Sciences Research Institute (ICVS), School of Medicine, University of Minho, Braga, Portugal. ${ }^{3}$ ICVS/3B's-PT, Government Associate Laboratory, 4710-057 Braga, Guimarães, Portugal.

Received: 24 June 2020 Accepted: 7 October 2020

Published online: 14 October 2020

\section{References}

1. Bharucha $A E$, Pemberton JH, Locke GR. American gastroenterological association technical review on constipation. Gastroenterology. 2013;144(1):218-38.

2. Bharucha $A E$, Rao SSC. An update on anorectal disorders for gastroenterologists. Gastroenterology. 2014;146(1):37-45.

3. Carrington EV, Scott SM, Bharucha A, et al. International anorectal physiology working group and the international working group for disorders of gastrointestinal motility and function. Expert consensus document: advances in the evaluation of anorectal function. Nat Rev Gastroenterol Hepatol. 2018;15(5):309-23.

4. Schmulson MJ, Drossman DA. What is new in Rome IV. J Neurogastroenterol Motil. 2017;23(2):151-63.

5. Simren M, Palsson OS, Whitehead WE. Update on Rome IV criteria for colorectal disorders: implications for clinical practice. Curr Gastroenterol Rep. 2017;19(4):15.

6. Chedid V, Vijayvargiya $\mathrm{P}$, Halawi $\mathrm{H}$, et al. Audit of the diagnosis of rectal evacuation disorders in chronic constipation. Neurogastroenterol Motil. 2019;31(1):e13510.
7. Videlock EJ, Lembo A, Cremonini F. Diagnostic testing for dyssynergic defecation in chronic constipation: meta-analysis. Neurogastroenterol Motil. 2013:25(6):509-20.

8. Dik VK, Siersema PD, Joseph A, et al. Constipation-related direct medical costs in 16887 patients newly diagnosed with chronic constipation. Eur J Gastroenterol Hepatol. 2014;26(11):1260-6.

9. Caetano AC, Santa-Cruz A, Rolanda C. Digital rectal examination and balloon expulsion test in the study of defecatory disorders: are they suitable as screening or excluding tests? Can J Gastroenterol Hepatol. 2016; ID 8654314.

10. Altomare DF, Spazzafumo L, Rinaldi M, et al. Set-up and statistical validation of a new scoring system for obstructed defaecation syndrome. Colorectal Dis. 2008;10(1):84-8.

11. Renzi A, Brillantino A, Di Sarno G, et al. Five-item score for obstructed defecation syndrome: study of validation. Surg Innov. 2013;20(2):119-25.

12. Minguez $M$, Herreros $B$, Sanchiz V, et al. Predictive value of the balloon expulsion test for excluding the diagnosis of pelvic floor dyssynergia in constipation. Gastroenterology. 2004;126(1):57-62.

13. Noelting J, Ratuapli SK, Bharucha AE, et al. Normal values for highresolution anorectal manometry in healthy women: effects of age and significance of rectoanal gradient. Am J Gastroenterol. 2012;107:1530-6.

14. Chiarioni G, Kim SM, Vantini I, et al. Validation of the balloon evacuation test: reproducibility and agreement with findings from anorectal manometry and electromyography. Clin Gastroenterol Hepatol. 2014;12(12):2049-54.

15. Talley NJ. How to do and interpret a rectal examination in gastroenterology. Am J Gastroenterol. 2008;103:820-2.

16. Orkin BA, Sinykin SB, Lloyd PC. The digital rectal examination scoring system (DRESS). Dis Colon Rectum. 2010;53(12):1656-60.

17. Bellini M, Usai-Satta P, Bove A, et al. Chronic constipation diagnosis and treatment evaluation: the "CHRO.CO.DI.T.E." study. BMC Gastroenterol. 2017;17(1):11.

18. Tantiphlachiva K, Rao P, Attaluri A, et al. Digital rectal examination is a useful tool for identifying patients with dyssynergia. Clin Gastroenterol Hepatol. 2010:8(11):955-60.

19. Caetano AC, Dias S, Santa-Cruz A, et al. Renzi score for obstructed defecation syndrome-validation of the portuguese version according to the cosmin checklist. Arq Gastroenterol. 2018;55(1):55-60.

20. Mokkink L, Terwee C, Patrick D. The COSMIN checklist manual. 2012: 1-56.

21. Beaton DE, Bombardier C, Guillemin F, et al. Guidelines for the process of cross-cultural adaptation of self-report measures. Spine (Phila Pa 1976). 2000:25(24):3186-91.

22. Rao SSC, Mudipalli RS, Stessman M, et al. Investigation of the utility of colorectal function tests and Rome II criteria in dyssynergic defecation (Anismus). Neurogastroenterol Motil. 2004;16(5):589-96.

23. Noelting J, Eaton JE, Choung RS, et al. The incidence rate and characteristics of clinically diagnosed defecatory disorders in the community. Neurogastroenterol Motil. 2016;28(11):1690-7.

24. Ramage L, Georgiou P, Qiu S, et al. Can we correlate pelvic floor dysfunction severity on MR defecography with patient-reported symptom severity? Updates Surg. 2018;70(4):467-76.

25. Eltringham MT, Khan U, Bain IM, et al. Functional defecation disorder as a clinical subgroup of chronic constipation: analysis of symptoms and physiological parameters. Scand J Gastroenterol. 2008;43(3):262-9.

26. Ratuapli S, Bharucha AE, Harvey D, Zinsmeister AR. Compariso of rectal balloon expulsion test in seated and left lateral positions. Neurogastroenterol Motil. 2013;25(12):e813-20.

\section{Publisher's Note}

Springer Nature remains neutral with regard to jurisdictional claims in published maps and institutional affiliations. 